\title{
Neighborhood disparities in stroke and myocardial infarction mortality: a GIS and spatial scan statistics approach
}

\author{
Ashley Pedigo', Tim Aldrich ${ }^{2}$ and Agricola Odoi ${ }^{*}$
}

\begin{abstract}
Background: Stroke and myocardial infarction (MI) are serious public health burdens in the US. These burdens vary by geographic location with the highest mortality risks reported in the southeastern US. While these disparities have been investigated at state and county levels, little is known regarding disparities in risk at lower levels of geography, such as neighborhoods. Therefore, the objective of this study was to investigate spatial patterns of stroke and MI mortality risks in the East Tennessee Appalachian Region so as to identify neighborhoods with the highest risks.

Methods: Stroke and MI mortality data for the period 1999-2007, obtained free of charge upon request from the Tennessee Department of Health, were aggregated to the census tract (neighborhood) level. Mortality risks were age-standardized by the direct method. To adjust for spatial autocorrelation, population heterogeneity, and variance instability, standardized risks were smoothed using Spatial Empirical Bayesian technique. Spatial clusters of high risks were identified using spatial scan statistics, with a discrete Poisson model adjusted for age and using a 5\% scanning window. Significance testing was performed using 999 Monte Carlo permutations. Logistic models were used to investigate neighborhood level socioeconomic and demographic predictors of the identified spatial clusters.

Results: There were 3,824 stroke deaths and 5,018 Ml deaths. Neighborhoods with significantly high mortality risks were identified. Annual stroke mortality risks ranged from 0 to 182 per 100,000 population (median: 55.6), while annual MI mortality risks ranged from 0 to 243 per 100,000 population (median: 65.5). Stroke and MI mortality risks exceeded the state risks of 67.5 and 85.5 in $28 \%$ and $32 \%$ of the neighborhoods, respectively. Six and ten significant $(p<0.001)$ spatial clusters of high risk of stroke and MI mortality were identified, respectively. Neighborhoods belonging to high risk clusters of stroke and Ml mortality tended to have high proportions of the population with low education attainment.
\end{abstract}

Conclusions: These methods for identifying disparities in mortality risks across neighborhoods are useful for identifying high risk communities and for guiding population health programs aimed at addressing health disparities and improving population health.

\section{Background}

On average, every 34 and 40 seconds, myocardial infarction (MI) and stroke events occur in the US, respectively [1]. Stroke ranks third in causes of death and is the leading cause of debilitation among Americans [2]. It is estimated that approximately $15 \%$ of those who have an

\footnotetext{
*Correspondence: aodoi@utk.edu

'The University of Tennessee, Department of Comparative Medicine, 2407

River Drive Knoxville, Tennessee 37996-4543, USA

Full list of author information is available at the end of the article
}

MI will die of it [1]. These health conditions are serious economic burdens to the US health system with annual costs estimated at $\$ 73.7$ billion for stroke and $\$ 177.1$ billion for MI [1].

Place of residence is an important determinant of cardiovascular health and disparities in the burdens of stroke and MI have been observed for different geographic areas [1-3]. The highest risks of mortality have been reported in the southeastern US [1,4-6] and in populations living in rural areas [7-9], particularly in the

\section{Biomed Central}

(c) 2011 Pedigo et al; licensee BioMed Central Ltd. This is an Open Access article distributed under the terms of the Creative Commons Attribution License (http://creativecommons.org/licenses/by/2.0), which permits unrestricted use, distribution, and reproduction in any medium, provided the original work is properly cited. 
Appalachian region [10,11]. Many areas of the Appalachian region, including parts of Tennessee, form a portion of the US "stroke belt". Tennessee ranks $3^{\text {rd }}$ highest in the US for stroke [1], and had an annual age-adjusted stroke mortality risk for the period 2000-2006 of 67.5 deaths per 100,000 persons compared to the national risk of 53.5 deaths per 100,000 persons [12]. For coronary heart disease including MI, Tennessee ranks $4^{\text {th }}$ highest in the US [1] with an annual age-adjusted mortality risk for the period 2000-2006 of 85.5 deaths per 100,000 persons compared to the national risk of 58.9 death per 100,000 persons [12]

The geographic distributions of stroke and MI mortality have been investigated at state and county levels $[1,5,11]$. However, geographic disparities have been shown to exist even after adjusting for variations in common risk factors like demographic factors (race, age), socioeconomic measures (income, education), behaviors (smoking, physical activity), and other conditions (diabetes, hypertension) $[4,10,11,13]$. These findings suggest that geographic variation in stroke and MI mortality could be due to more localized distributions of neighborhood risk factors. The clustering of determinants of stroke and MI at the neighborhood level can greatly affect the planning, implementation, and focus of health initiatives that seek to reduce disparities. Therefore, research should focus on identifying disparities at the neighborhood level to better understand health needs and thus, provide needs-based health services $[3,14]$. While many studies have defined neighborhoods as census tracts or smaller geographic units, the neighborhoods have not been used as the unit of analysis for many past studies investigating cardiovascular disease and stroke [15-21]. Rather, these studies have investigated neighborhood characteristics as contextual effects in multilevel models that seek to explain individual level risk. Thus, ecological studies are needed to investigate the spatial patterns and clustering of high mortality risk with the neighborhood as the unit of analysis since this is important in identifying high risk communities and targeting resources to address health disparities and improve population health at the local level.

When investigating disease patterns in small geographic areas like neighborhoods, however, there are some challenges that must be addressed. Due to population heterogeneity, mortality risks from areas of low population will likely have higher variances and therefore be more unstable than those from areas of high population [22]. This variance instability of small geographic areas is referred to as the small number problem [23]. Spatial smoothing of risks is used to mitigate this issue by reducing the "noise" from areas with low population and therefore high variances [24].
With these issues in mind, the objective of this study was to investigate spatial patterns and detect local neighborhood clusters of high risk of stroke and MI mortality in the East Tennessee Appalachian Region. The identification of neighborhoods with high risks is expected to aid local health planners in understanding the specific neighborhood health needs to guide health planning and provision of health services. Thus, identified clusters of high risks of stroke and MI mortality will be useful in guiding resource allocation, service provision, and policy decisions at the local/neighborhood level that are crucial for addressing neighborhood health disparities.

\section{Methods}

\section{Study area and data collection}

The study area included eleven counties of the East Tennessee Appalachian Region that have some of the highest risks of stroke and/or MI in the state: Claiborne, Cocke, Grainger, Greene, Hamblen, Hancock, Hawkins, Jefferson, Knox, Sevier, and Union counties. This area had a population of just over 780,000 persons in 2000 and included 168 census tracts. Census tracts (CTs) are statistical subdivisions of a county that have between 2,500 and 8,000 persons, do not cross county boundaries, and are homogenous with respect to population characteristics, economic status, and living conditions [25]. Since they are good proxies of natural neighborhood boundaries and are therefore useful in describing neighborhood population characteristics and health disparities [26,27], CTs were chosen as the geographical unit of analysis and were used to represent neighborhoods in this study.

Mortality data from 1999 to 2007 were obtained free of charge, upon request, from the Tennessee Department of Health. Thus, although these data are freely available on request from the responsible authorities, they are not currently openly available for internet downloads. Stroke and MI deaths were identified by ICD 10 codes I60-I69 and I21-I22, respectively. For the 8,842 mortality records obtained, complete street address data were available for $94 \%$, while the other $6 \%$ had missing or inadequate (such as post office box) address data. The addresses were geo-coded using BatchGeo [28], an online geo-coding service which implements the Google Maps geocoding application programming interface (API) that has some of the highest quality geocoding databases available $[29,30]$. Exact, or roof top, address matches were obtained for $67 \%$ of the data, while $30 \%$ were range interpolated between two points on the street and $3 \%$ were matched to the zipcode. The geographic coordinates were imported into ArcGIS 9.3 [31] where point-in-polygon join was used to link the mortality data to the openly available census 
tract level cartographic boundary files downloaded from the U.S. Census Bureau website [32].

Census tract level socioeconomic, demographic, and population data for the study area were obtained from the openly available census 2000 summary file 3 [33]. Since these data are available in the US only through the decennial census, the 2000 data was deemed best suited to match the disease data (1999-2007). The neighborhood variables chosen to be assessed as potential predictors of the geographic distribution of MI and stroke high risk mortality clusters were based on current knowledge in the literature. They include: black race $[3,5,8,34]$, gender $[2,8,35,36]$, age 65 years and older $[2,8,15,37]$, household income $[15,16,18,21,38]$, education less than high school $[8,21,39,40]$, population below poverty $[16,21,41]$, median housing value $[39,42,43]$, geography (urban versus rural) $[3,7,8,44]$, and factors like employment, single parent families, marital status, and housing ownership that have been used in composite measures of socioeconomic status (SES) or deprivation $[39,42,43,45]$.

\section{Data analysis}

\section{Data management}

One neighborhood in Knox county, that had a population of 232 and included a mental health facility, was removed from the analysis due to missing data values for most of the variables. With the exception of median household income, median housing value, and family size, all variables were analyzed as the proportion of the population in each CT (neighborhood).

\section{Descriptive analyses, risk standardization and spatial smoothing}

All descriptive analyses were done in SAS 9.2 [46]. Significance of the difference in median age between genders was assessed using the Wilcoxon rank sum test [47]. Mortality risks for neighborhoods were ageadjusted using direct standardization in Stata 11 [48]. All risks were expressed as the annual number of deaths per 100,000 population.

The raw (unsmoothed) age-adjusted risks were expected to have high variances due to the small number problem since there were areas of low population and some neighborhoods with only a few cases of stroke/MI in the study area [23]. To address this issue, as well as adjust for spatial autocorrelation and population heterogeneity, the raw age-adjusted risks were smoothed using Spatial Empirical Bayes (SEB) smoothing using $2^{\text {nd }}$ order queen weights in GeoDa [49]. In this smoothing method, the risks for low population neighborhoods in areas without clear spatial patterns are shrunk toward the global mean of the study area $[22,50]$. Conversely, in areas where obvious spatial patterns exist, the less reliable estimates from low population areas are adjusted towards a local mean. Thus, the SEB smoothed risks are more stable than raw (unsmoothed) risks [24].

\section{Detection and identification of stroke and MI clusters}

To detect the presence of high risk stroke and MI clusters and identify their locations, the spatial scan statistic, implemented in SaTScan, was used [51]. The technique uses circular windows of variable radius that move across the study area to compare the number of deaths in the window with what would be expected if the deaths were distributed randomly in space [51]. The window radius varies from zero up to a specified maximum. Each window defines a set of different neighboring CTs, such that if the geographic centroid of a CT is contained in the window, then the deaths and population from that whole CT are included. Clusters are identified based on a likelihood ratio test [52] with a p-value obtained using Monte Carlo replications [53]. The primary cluster, with the highest significant likelihood, is interpreted such that there is an increased risk of stroke/MI mortality within the window compared to outside [54].

Non-overlapping, spatial clusters of high risk of stroke/MI mortality were identified using a purely spatial, discrete Poisson model [52] adjusted for age distribution. Since the results of this analysis can be sensitive to model parameters, particularly window size, care must be taken in its choice. The goal of the current analyses was to identify local clusters of high mortality risks among neighborhoods. Thus, similar to another study [55], the window size of $5 \%$ of the total population was chosen based on the population of the largest neighborhood so that potentially one single neighborhood could constitute a distinct high risk cluster.

\section{Logistic modeling of predictors of high risk stroke or MI clusters}

The outcome of interest in this modeling was binary, reflecting whether a neighborhood belonged to a cluster or not. Univariate associations of continuous variables with the outcomes were assessed using Wilcoxon rank sum test for non-normally distributed data, while chisquare and exact tests were used for categorical variables. Variables with significant associations based on a liberal $\mathrm{p}$-value $(\mathrm{p}=0.20)$ were considered in the modeling process along with some non-significant variables that had been shown in literature to be strongly associated with the outcome.

Multiple logistic models were used to investigate potential associations between log odds of a neighborhood being in a high risk stroke or MI cluster and a number of neighborhood level socioeconomic and demographic characteristics. The assumption of linearity of continuous variables with the log odds of the outcome (belonging to a stroke or MI cluster) for logistic modeling were assessed using graphical methods. Only 
the proportions of the population $\geq 65$ years and of single parent families met this assumption for stroke cluster, while the proportions of population with less than high school education, those living below poverty and median housing value met the assumption for the MI outcome. Therefore, these variables were modeled as continuous variables. The variables not meeting the linearity assumption were transformed into categorical variables using either a priori considerations or quartile cutpoints from the distribution of the variable.

The model was built by starting with the full model and then removing variables based on the following criteria: (1) the highest non-significant $\mathrm{p}$-value (with significance set to $\mathrm{p}=0.05)$; (2) a likelihood ratio test of the model with and without the variable that was non-significant; and (3) the variable was not an important confounder of other variables in the model. Variables were considered important confounders if their removal from the model resulted in a large (greater than 20\%) change in the coefficients of any of the remaining variables in the model. Categorical variables were analyzed as regular dummy variables. The significance in the model of each group of dummy variables (belonging to one categorical variable) was analyzed using a likelihood ratio test. Two-way interaction terms between gender, race, age, income, education, poverty, and geography were assessed for statistical significance $[8,35,45,56]$. Model fits were assessed using the Pearson and Hosmer-Lemeshow goodness of fit tests and residual diagnostics. The predictive abilities of the models were evaluated using sensitivity, specificity, and overall correct classifications.

\section{Cartographic displays}

All cartographic manipulations and displays were done in ArcGIS 9.3 [31]. The intervals for displaying the ageadjusted SEB smoothed mortality risks of stroke and MI in the choropleth maps were determined using Jenk's optimization classification scheme. Since SEB risks are more appropriate for mapping in small areas compared to unsmoothed risks [23,24], only the former are presented. Significant spatial clusters were displayed in ArcGIS 9.3 [31].

\section{Results}

\section{Description of stroke and MI deaths}

There were 3,824 stroke deaths in the study area from 1999 to 2007. No stroke deaths were reported in 18 of the 168 neighborhoods. Women accounted for 2,435 (63.7\%) of the stroke deaths. The median age was significantly ( $\mathrm{p}<0.001$ ) lower for men (median 78; range 4103), than women (median 81; range 3-103). Persons dying from stroke or MI in the study were primarily white $(94 \%)$ and had less than a high school education $(45 \%)$. It is worth noting that $92 \%$ of the population in the study area was white, while $25 \%$ of the population older than 18 years had less than high school education.

Myocardial infarction was the cause of 5,018 deaths during the study period. No deaths were reported in 17 neighborhoods; 15 of these neighborhoods also had no reported stroke deaths. More MI deaths occurred in men (2,745 deaths, 54.6\%) than women (45.4\%). Again, the median age of death was significantly $(\mathrm{p}<0.001)$ lower for men (median 71; range 21-102), than women (median 81; 27-106).

\section{Spatial distribution of mortality risks Stroke risks}

The annual median age-adjusted raw (unsmoothed) stroke risk for the study area was 55.6 deaths/100,000 population (range: $0-182$ ), with $28 \%$ of the neighborhoods exceeding the state stroke mortality risk of 67.5 [12]. Similarly, the annual median SEB smoothed stroke risk was 56.1 deaths/100,000 population (range: 0.1 174). The annual median risk for the study area remained constant from 1999 to 2007. The highest stroke risks (greater than 110 deaths/100,000) were observed in three neighborhoods in Knox county and one neighborhood each in Jefferson and Hamblen counties (Figure 1). It appeared that the neighborhoods with stroke risks higher than the state risk were concentrated across neighborhoods in the northwest portions of Cocke and Greene counties, in addition to a few neighborhoods in Grainger, Hamblen, and Jefferson counties, as well as in the downtown area of Knox county. These neighborhoods are primarily located in or near city centers in the study area.

\section{Myocardial infarction risks}

The annual median raw (unsmoothed) age-adjusted MI mortality risk was 65.5 deaths/100,000 population (range: 0-243), while the median SEB smoothed risk was 63.5 (range: 0.5-235). Myocardial infarction mortality risks in the study area were higher than the state risk of 85.5 [12] in $32 \%$ of the neighborhoods. The spatial distribution of neighborhood risks revealed patterns of high risks across the study area (Figure 2). The areas with the highest MI risks (greater than 140 deaths/ $100,000)$ included all neighborhoods in Claiborne county and all but one neighborhood in Cocke county. In addition to these counties, neighborhoods with risks above the state risk were also located in Greene, Jefferson, Hamblen, Grainger, and Knox counties in a pattern very similar to that for stroke risks.

\section{Spatial clusters of high stroke/MI mortality risks}

Table 1 displays results of identified significant spatial stroke and MI mortality clusters. For each cluster, the table gives the number of census tracts in the cluster, the total population, the observed number of stroke or 


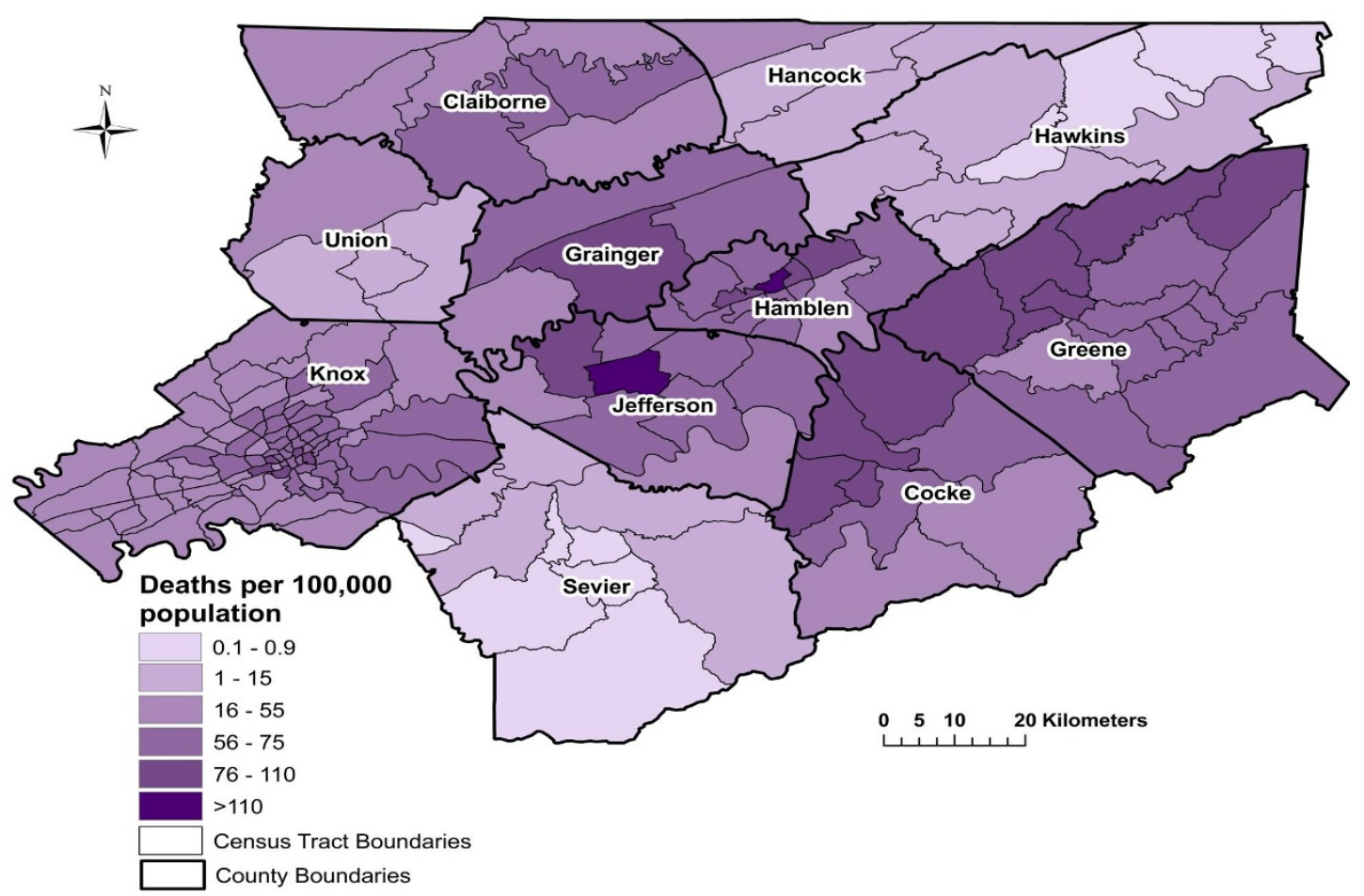

Figure 1 Spatial Empirical Bayes smoothed age-adjusted stroke mortality risk per 100,000 population from 1999 to 2007 in East Tennessee Appalachian region.

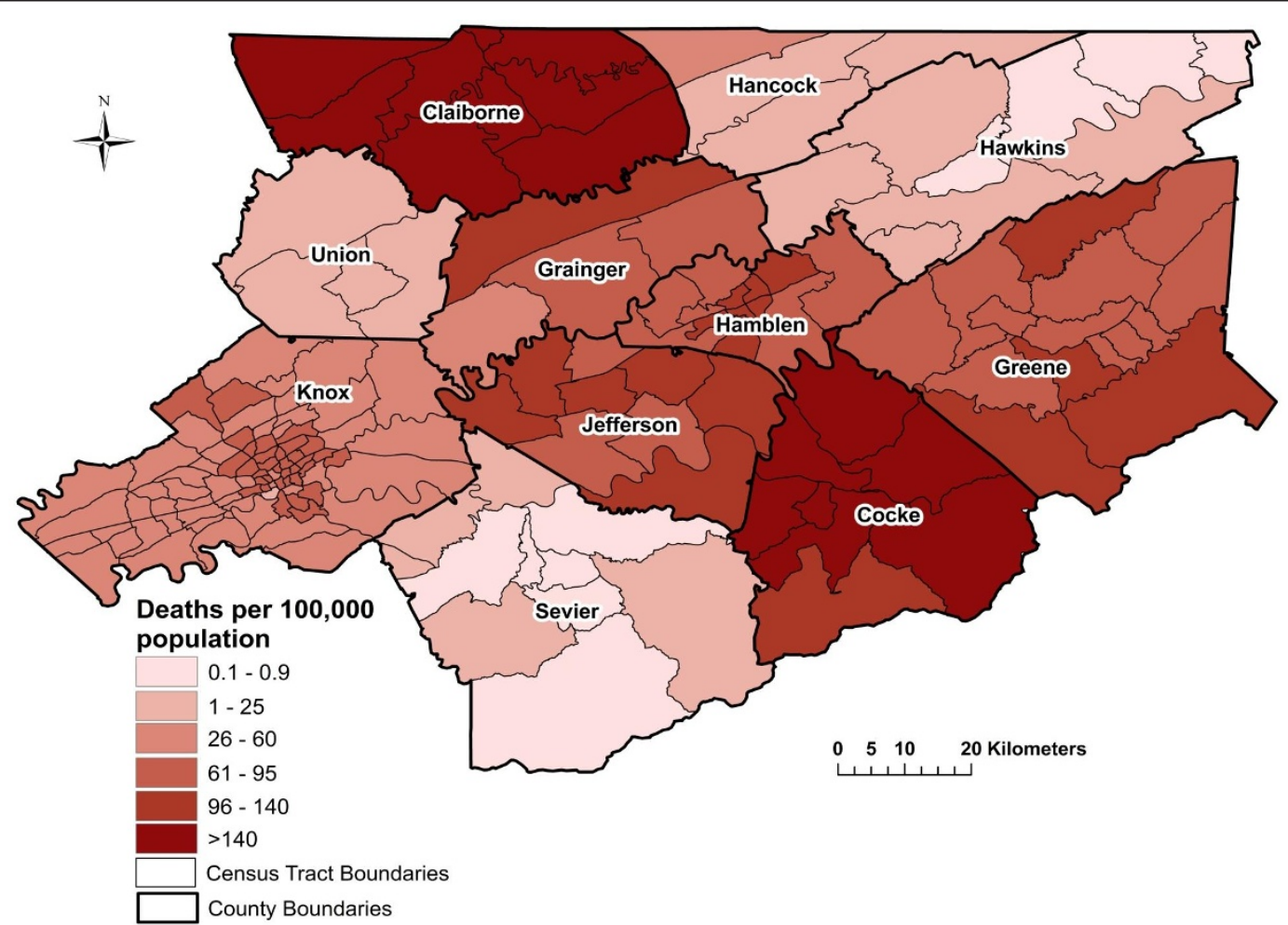

Figure 2 Spatial Empirical Bayes smoothed age-adjusted myocardial infarction mortality risk per 100,000 population from 1999 to 2007 in East Tennessee Appalachian region. 
Table 1 Spatial clusters of age-adjusted stroke and myocardial infarction mortality risks from 1999 to 2007 in East Tennessee Appalachian region

\begin{tabular}{|c|c|c|c|c|c|c|}
\hline Cluster & $\begin{array}{l}\text { \# of Census Tracts } \\
\text { (Neighborhoods) }\end{array}$ & Population & Observed \# of Deaths & Expected \# of Deaths & $\begin{array}{c}\text { Annual \# of } \\
\text { Deaths/100,000 Persons }\end{array}$ & P-value \\
\hline \multicolumn{7}{|c|}{ Stroke } \\
\hline 1 & 1 & 5,447 & 136 & 37.76 & 195.6 & 0.001 \\
\hline 2 & 3 & 17,243 & 174 & 91.95 & 102.8 & 0.001 \\
\hline 3 & 6 & 34,887 & 270 & 174.24 & 84.2 & 0.001 \\
\hline 4 & 6 & 30,158 & 266 & 187.63 & 77.0 & 0.001 \\
\hline 5 & 5 & 24,711 & 180 & 120.65 & 81.0 & 0.001 \\
\hline 6 & 4 & 12,008 & 107 & 67.27 & 86.4 & 0.004 \\
\hline \multicolumn{7}{|c|}{ Myocardial Infarction } \\
\hline 1 & 7 & 36,945 & 608 & 243.77 & 177.7 & 0.001 \\
\hline 2 & 6 & 24,596 & 334 & 159.80 & 148.9 & 0.001 \\
\hline 3 & 4 & 13,856 & 213 & 88.78 & 171.0 & 0.001 \\
\hline 4 & 6 & 28,823 & 333 & 197.74 & 120.0 & 0.001 \\
\hline 5 & 6 & 30,158 & 363 & 236.98 & 109.2 & 0.001 \\
\hline 6 & 3 & 9,568 & 124 & 61.61 & 143.4 & 0.001 \\
\hline 7 & 7 & 35,548 & 325 & 231.94 & 99.9 & 0.001 \\
\hline 8 & 1 & 2,818 & 47 & 20.94 & 160.0 & 0.001 \\
\hline 9 & 4 & 8,566 & 88 & 54.98 & 114.1 & 0.009 \\
\hline
\end{tabular}

\#: Number.

MI deaths in the cluster area, the expected number of deaths based on the Poisson model, the estimated annual number of cases per 100,000 persons, and the significance level ( $\mathrm{p}$-value) obtained from the likelihood ratio test with Monte Carlo permutations. Figures 3 and 4 display geographic distributions of the significant spatial clusters of stroke and MI, respectively.

\section{Stroke clusters}

Six significant $(\mathrm{p}<0.001)$ spatial clusters of high risk of stroke mortality were identified (Table 1 and Figure 3). The smallest cluster, which was also the primary cluster, was comprised of only 1 neighborhood in Hamblen county. The relative risk of this cluster was 3.7 (Figure 3 ), implying that the risk of death from stroke was 3.7 times higher within cluster 1 than other neighborhoods in the study area. Relative risks for the secondary clusters ranged from 1.5 to 1.9. Cluster 3 accounted for the highest number of stroke deaths and was composed of 6 neighborhoods in Cocke and Hamblen counties. The second largest cluster (cluster 4) included 6 neighborhoods in Greene county. The majority of the high risk stroke clusters were located in or near city centers.

\section{Myocardial infarction clusters}

There were nine significant $(\mathrm{p}<0.009)$ spatial clusters of high risk of MI mortality (Table 1 and Figure 4). The primary cluster was the largest cluster in both the number of MI deaths and geographic size, and included neighborhoods in Cocke and Hamblen counties. The populations in cluster 1 neighborhoods had a risk of death from MI that was 2.7 times greater than other neighborhoods in the study area. Relative risks for the secondary clusters ranged from 1.4 to 2.5 . Cluster 7 was the second largest and included neighborhoods in Jefferson, Hamblen, and Grainger counties. Neighborhoods in Claiborne, Greene, and Knox counties were also parts of significant high risk MI clusters. The majority (76\%) of neighborhoods in significant high risk stroke clusters also belonged to significant high risk MI clusters.

\section{Predictors of high risk stroke and myocardial infarction spatial clusters \\ Stroke}

The univariate associations of the socioeconomic and demographic variables of interest with the outcome of belonging to a high risk stroke cluster are presented in Table 2. Variables with significant associations, based on a liberal $\mathrm{p}$-value $=0.20$ were further assessed in the multivariable logistic model. Even though gender was non-significant it was included because disparities in stroke risk and mortality by gender have been reported in literature $[2,8,40,41]$. The other non-significant variables were not included because they were each highly correlated $(r>0.70)$ with median household income. The final model had a highly significant $(\mathrm{p}=0.0002)$ likelihood. The proportion of the population with less than a high school education $(\mathrm{p}=0.015)$ and that were black $(\mathrm{p}=0.019)$ were significant variables in the model (Table 3). Neighborhood geography (rural, suburban, urban) was not significant ( $\mathrm{p}=0.1$ ), but was included in the final model because it was an important confounder 


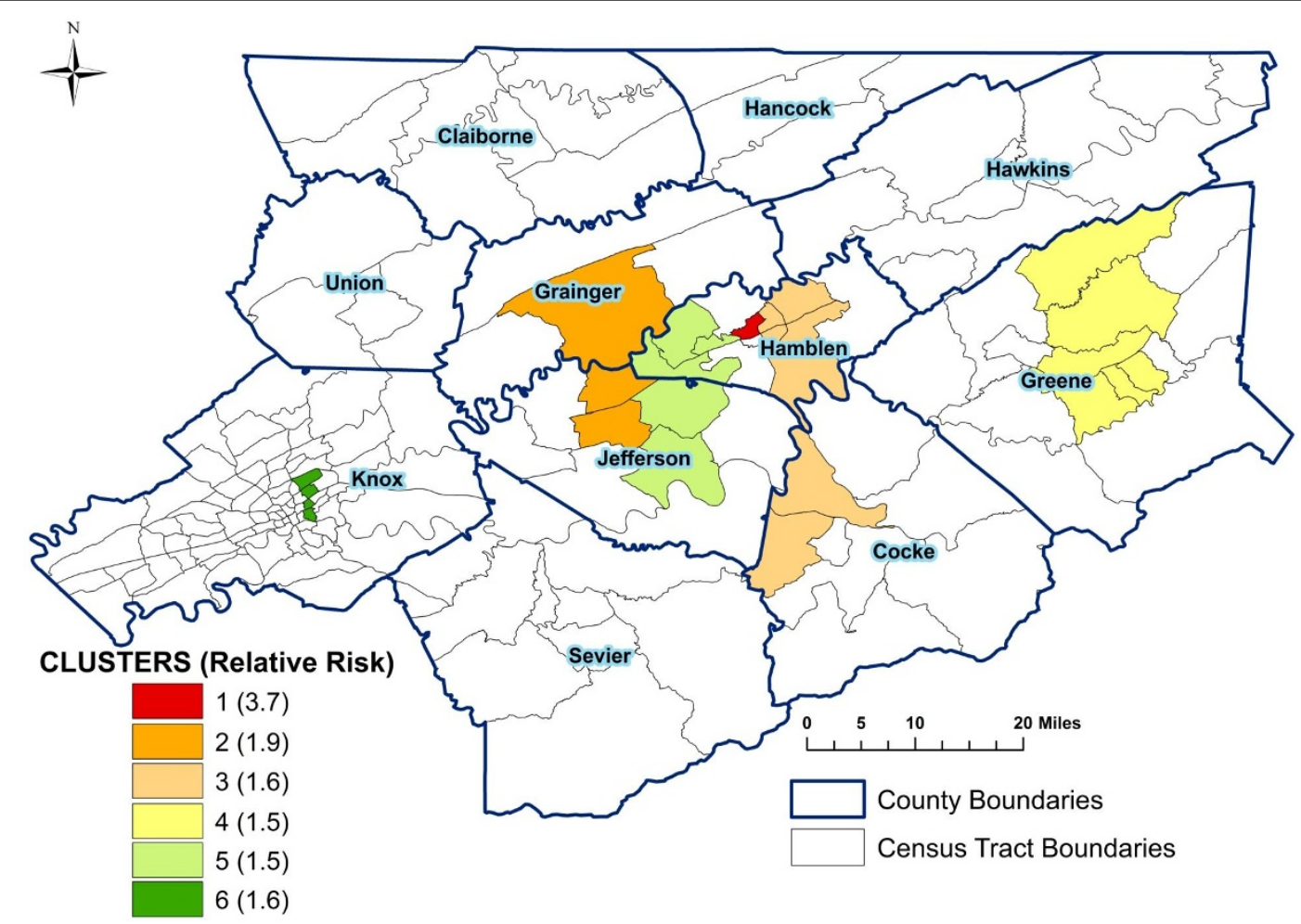

Figure 3 Significant spatial clusters of high age-adjusted stroke mortality risks from 1999 to 2007 in East Tennessee Appalachian region.

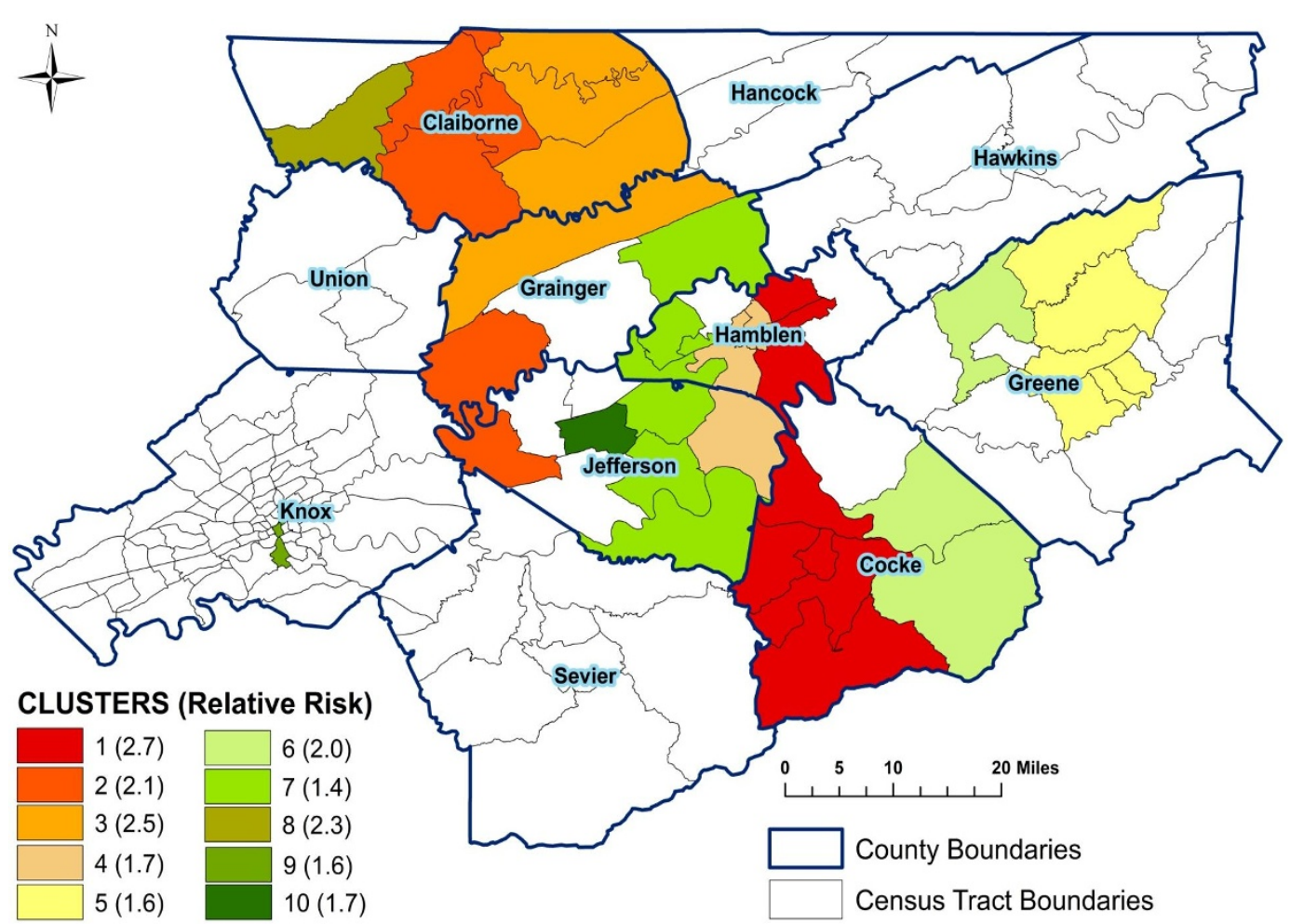

Figure 4 Significant spatial clusters of high age-adjusted myocardial infarction mortality risks from 1999 to 2007 in East Tennessee Appalachian region. 
Table 2 Univariate associations of high risk stroke mortality clusters with neighborhood socioeconomic and demographic factors

\begin{tabular}{|c|c|c|}
\hline \multirow[t]{2}{*}{ Neighborhood level socioeconomic and demographic variables } & \multicolumn{2}{|c|}{ Significance value } \\
\hline & Stroke cluster & MI cluster \\
\hline Geography (rural, suburban, urban) & $0.10^{\S}$ & $0.02^{\S}$ \\
\hline Proportion of black population & $0.02^{\S}$ & $0.58^{\S}$ \\
\hline Proportion population age $\geq 65$ years & $0.02^{\S}$ & $0.19^{\S}$ \\
\hline Proportion of single parent families & $0.04^{\S}$ & $0.08^{\S}$ \\
\hline Proportion of owner occupied housing units & $0.08^{\S}$ & $0.02^{\S}$ \\
\hline Median household income (\$) & $0.15^{\S}$ & $0.03^{\S}$ \\
\hline Proportion of population with $<$ high school education & $0.18^{\S}$ & $0.00^{\S}$ \\
\hline Proportion of married persons & $0.20^{\S}$ & 0.36 \\
\hline Average family size & 0.34 & 0.27 \\
\hline Proportion of population living below poverty & 0.41 & $0.00^{\S}$ \\
\hline Median housing value $(\mathrm{S})$ & 0.41 & $0.00^{\S}$ \\
\hline Gender & $0.60^{\S}$ & $0.04^{\S}$ \\
\hline Proportion of population employed & 0.67 & $0.00^{\S}$ \\
\hline
\end{tabular}

\footnotetext{
${ }^{\S}$ Variables assessed in subsequent multivariable logistic regression model.
}

of race such that its removal resulted in a $30 \%$ change for coefficients for proportion of blacks. No interaction terms were significant at the $\mathrm{p}<0.05$ level. Neighborhoods with higher proportion of population with less than a high school education had significantly higher odds of belonging to a stroke cluster compared to those with low proportion of the population with less than high school education.

Goodness of fit tests showed no evidence $(\mathrm{p}=0.389)$ that the model was not fitting the data well. The model had very high specificity (97.8\%) (i.e. the ability to correctly predict no cluster given the neighborhood was not in a cluster). However, it had a relatively low
(20\%) sensitivity (i.e. the ability to predict being in a stroke cluster given that the neighborhood was truly in a cluster). The positive predictive value, or the probability of being in a cluster given the model predicted cluster, was $62.5 \%$. The negative predictive value, or the probability of not being in a cluster given that the model predicted no cluster, was $87.4 \%$. Overall, the model has a correct classification rate of $86.2 \%$. There were a few outliers, with large positive residuals in the model. These neighborhoods were primarily urban, with the lowest proportion of population of blacks, and the lowest levels of population without high school education.

Table 3 Final logistic model showing socioeconomic and demographic predictors of high risk stroke mortality clusters

\begin{tabular}{|c|c|c|c|}
\hline Variable & Coefficient & $\begin{array}{c}\text { LRT* } \\
\text { p-value }\end{array}$ & 95\% Confidence Interval \\
\hline Constant & -6.036 & & $-8.467,-3.605$ \\
\hline Geography & & 0.17 & \\
\hline Rural & Referent & & - \\
\hline Suburban & 1.299 & & $-0.170,2.769$ \\
\hline Urban & 1.351 & & $-0.340,3.042$ \\
\hline Proportion of Blacks & & 0.02 & \\
\hline$<0.02$ & Referent & & - \\
\hline$>0.02-\leq 0.05$ & 1.179 & & $-0.127,2.486$ \\
\hline$>0.05-\leq 0.10$ & 1.631 & & $-0.095,3.357$ \\
\hline$>0.10$ & -0.629 & & $-2.589,1.35$ \\
\hline Proportion of Pop with < High School education & & 0.02 & \\
\hline$\leq 0.17$ & Referent & & - \\
\hline$>0.17-\leq 0.30$ & 2.913 & & $0.699,5.127$ \\
\hline$>0.30-\leq 0.37$ & 3.022 & & $0.740,5.304$ \\
\hline$>0.37$ & 3.898 & & $1.527,6.268$ \\
\hline
\end{tabular}

*LRT (Likelihood ratio test) p-value = test of significance of each group of dummy variables (belonging to one categorical variable). Thus, this tests the statistical significance of the variable as a whole (all parameter estimates of the categories of variable in the model). 


\section{Myocardial infarction}

The univariate associations of the socioeconomic and demographic variables of interest with the outcome of belonging to a high risk MI mortality cluster are presented in Table 2. Variables with significant associations, based on a liberal p-value $=0.20$ were further assessed in the multivariable logistic model. The proportion of the neighborhood population of blacks was non-significant, but it was included in the analyses because disparities in MI risk and mortality by race have been reported in the literature $[3,5,8,34]$. The final model, based on the prescribed criteria for removal of variables, had a highly significant likelihood $(\mathrm{p}<0,001)$ (Table 4). The proportion of the population with less than high school education, modeled as a continuous variable, was the strongest predictor of the odds of being in a MI cluster. Geography $(\mathrm{p}=0.05)$ and gender $(\mathrm{p}$ $=0.03$ ) were significant based on the likelihood ratio test of their respective dummy variables as a group. Suburban and urban neighborhoods had significantly higher odds of belonging to an MI cluster compared to rural neighborhoods. Neighborhoods with a higher proportion of males versus females also had higher odds of being in a cluster. The proportion of the population of black race was not significant $(\mathrm{p}=0.1$ ), but was included in the final model because it was an important confounder for both geography and gender such that its removal resulted in a more than $20 \%$ change for their coefficients. No interaction terms were significant at the $\mathrm{p}<0.05$ level.

Goodness of fit tests showed no evidence $(\mathrm{p}=0.521)$ that the model was not fitting the data well. The model had very high specificity $(90.2 \%)$. However, it had a relatively low (51.1\%) sensitivity. The positive predictive value was $65.7 \%$ while the. negative predictive value was
83.3\%. Overall, the model had a correct classification rate of $80 \%$. There were only three neighborhoods that the model did not fit well. These were rural neighborhoods that had the most extreme high levels of the proportions of the population without high school education.

\section{Discussion}

The results show that spatial patterns of high risk of stroke and MI exist in the study area. These findings are consistent with those from other studies that have reported that southern states like Tennessee $[1,6,9,34,44]$, and specifically Appalachian counties $[10,11,57]$, have excess risk of stroke and MI. The excess risk has mostly been attributed to variations in the distribution of stroke and MI risk factors such as race, socioeconomic status, geography (urban vs. rural), and prevalence of other chronic diseases, such as diabetes and hypertension $[3,6,9,58]$. However, other studies have reported that geographic disparities exist even after adjusting for variations in these risk factors $[4,10,11,13]$. The apparent inconsistency in the association between high risks of stroke/MI and risk factors at the state and county levels suggests that disparities may be due to more localized distributions of risk factors.

To our knowledge, this is the first study to investigate spatial patterns and clusters of stroke and MI risk to better understand observed disparities and identify specific health needs at the neighborhood level to aid population health planning. The results of the current study provide evidence that the risk of stroke and MI can be highly variable within a county and therefore studies that perform analyses at the county level fail to identify

Table 4 Final logistic model showing socioeconomic and demographic predictors of high risk myocardial infarction mortality clusters

\begin{tabular}{|c|c|c|c|}
\hline Variable & Coefficient & $\begin{array}{c}\text { LRT* }^{*} \\
\text { p-value }\end{array}$ & 95\% Confidence Interval \\
\hline Constant & -6.541 & & $-8.865,-4.220$ \\
\hline Proportion of Pop with < High School education & 14.562 & & $8.963,20.610$ \\
\hline Geography & & 0.05 & \\
\hline Rural & Referent & & - \\
\hline Suburban & 1.558 & & $0.205,2.911$ \\
\hline Urban & 1.544 & & $-0.033,3.122$ \\
\hline Proportion of Blacks & & 0.14 & \\
\hline$<0.02$ & Referent & & - \\
\hline$>0.02-\leq 0.05$ & 0.306 & & $-0.844,1.456$ \\
\hline$>0.05-\leq 0.10$ & -0.991 & & $-2.950,0.968$ \\
\hline$>0.10$ & -1.494 & & $-3.231,0.244$ \\
\hline Gender & & 0.03 & \\
\hline Proportion of Male Population $\leq 0.50$ & Referent & & - \\
\hline Proportion of Male Population > 0.50 & 1.024 & & $0.086,1.962$ \\
\hline
\end{tabular}

LRT (Likelihood ratio test) p-value = test of significance of each group of dummy variables (belonging to one categorical variable). Thus, this tests the statistical significance of the variable as a whole (all parameter estimates of the categories of variable in the model). 
these disparities at lower (neighborhood) levels. For example, Knox and Hamblen counties are often reported to have lower risks of stroke and MI and are not considered economically distressed/disadvantaged when compared to other counties in the area $[10,11]$. However, it is evident from the findings here that a few neighborhoods in these counties have very high risks and are part of significant spatial clusters for stroke and MI. If analyses, research, and planning activities to address disparities in risk are conducted at county or higher levels as is often done, these spatial disparities within the counties would be missed. Therefore, neighborhoods would likely be erroneously ignored in programs geared towards addressing disparities in MI and stroke risk. The implication is that for health research and planning activities to be most effective, the focus must be on neighborhood level characteristics and specific needs to alleviate the variation seen at higher geographic levels.

Other studies have used multilevel analyses, including both neighborhood and individual characteristics, to describe disparities in MI risk for individuals [15-21]. One study, using data from the Atherosclerosis Risk in Communities Study, categorized neighborhoods (CTs) into tertiles by neighborhood median household income and found that greater incidence risk of MI was associated with living in lower income neighborhoods [38]. Diez Rouz, et al. (2001) also found that living in a disadvantaged neighborhood was associated with increased incidence of coronary heart disease, including MI, while adjusting for individual income, education, and occupation and defining neighborhoods as census block groups [18]. However, some differences in incidence remained between neighborhoods after adjusting for common socioeconomic factors. The failure of individual level risk factors to substantially explain risk at aggregated levels is a common finding in multilevel studies [45]. Some authors have suggested that neighborhood level socioeconomic variables capture information above and beyond the individual level, and so do not serve only as proxies for individual risk factors [21]. Similar to reports from other studies $[16,21]$, we found that neighborhoods with a high proportion of the population with low education had higher stroke and MI risks. However, we did not find significant association between median household income and risk of MI or stroke. This is contrary to findings from previous studies $[15,18,38,43]$ and is likely because these were individual level studies while ours is a population/group (neighborhood) level study. In addition to the level of education, the confounding identified between the geography (urban versus rural), race, and gender distribution of each neighborhood is potentially important to understanding how geographic disparities arise in the study area. The influence of neighborhood socioeconomic and social conditions on health may be related, in part, to availability and accessibility to health care services, the built environment and infrastructure (i.e. quality schools, recreational facilities, stores and restaurants with healthy foods), neighborhood based attitudes towards health and related behaviors (i.e. smoking, physical activity, and diet), and the degree of social support $[14,20,59,60]$. Since health planning is performed at the population level, identifying geographic disparities for neighborhoods can provide insight into the social conditions, structures, and mechanisms that influence health outcomes in the population to better provide effective population based education campaigns and prevention strategies. Thus, studies, such as this one, that investigate neighborhood level patterns in risk should be considered in addition to those multilevel studies that assess risk of individuals in neighborhoods to ensure community health resources, services, and other efforts are best targeted to the populations at greatest risk.

Although mortality data are useful and commonly used in epidemiological studies to assess health and its patterns, they are not without limitations. First, the accuracy of the cause of death given on a death certificate can be affected by errors made by physicians or in coding, differences in diagnostic criteria, issues arising when there are multiple causes of death, or errors in data entry [61]. Lloyd-Jones et al. (1998) reported that death certificates overrepresented coronary heart disease as cause of death, particularly for older populations, and cautioned that its use in etiologic studies could potentially lead to a bias towards the null value [62]. There is also concern that mortality data reflects past, rather than current, health needs. However, mortality is often the most commonly available data for observational, population-based studies since (in the US) it is freely available through organizations, like health departments and the Centers for Disease Control and Prevention [61]. Unfortunately, the mortality data in this study contained only decedent's residential address for geo-coding to the census tract level and gave no information on whether the address was a place other than a private home, such as nursing homes or prisons, thus limiting the ability to assess any effect such issues would have on the results of the study. However, we did identify to the best of our ability, the addresses known to be nursing homes and found that no more than 15 deaths occurred at any given address. Thus, we do not believe these issues would significantly affect the spatial patterns observed.

From a methodological standpoint, while neighborhood level analyses provide the advantage of better insight and understanding of health disparities and needs, they are not without limitations. Due to the small 
number problem, visualization of raw risks from areas with low population or small number of deaths can be misleading. In this study, this problem was overcome using SEB smoothing of risks that reduces noise associated with population heterogeneity and variance instability by borrowing strength from neighbors. While the removal of noise from low populations with unstable risks eases visual interpretation, it may possibly introduce artifacts into the map [24,63] and therefore these risks should only be used for visualization and not statistical analyses [64,65]. Additionally, many smoothing techniques, including the SEB used in this study, are prone to edge effects such that neighborhoods on the edges of the study area have fewer neighbors than those in the interior, so there is less information to borrow from neighbors in smoothing [23]. Thus the risks are shrunk toward a global instead of the local mean. Despite these disadvantages, spatial smoothing of risks minimizes erroneous visual interpretations associated with raw risks by reducing noise, making spatial patterns more evident, and reducing attention to outliers by focusing on the overall geographic pattern of the study area [23]. In this study, the smoothed risks did not change the raw pattern very much, except to make localized patterns more visually obvious for both stroke and MI. This result indicates that extreme values (very high and low risks) in the wide mortality risk range were composed of neighborhoods with stable risks, i.e. risks with low variance. Since the SEB has a larger impact on unstable risks and little to no impact on stable risks (i.e. those with low variances) $[23,64]$, it is not unexpected that there were minimal differences between the raw (unsmoothed) and SEB risks.

The visual interpretation of spatial patterns can be strongly affected by the number and width of class intervals used to represent risk values $[23,66]$. To reduce this potential bias, it has been suggested that intervals should be based on the overall shape of the distribution and not statistical frequency [66]. Thus, this study employed the Jenks, or natural breaks, classification method which defines intervals based on the natural distribution of breaks or groupings in the data [67]. The visualization of spatial patterns of disease is an important component in identifying geographic disparities. However, it is standard epidemiology practice not to rely on one's visual interpretation of a map of disease risks to differentiate significant spatial clusters from what may seem to be a cluster visually but is not statistically significant $[24,65]$. Furthermore, interpretations of spatial patterns from visual investigations become even more difficult when the population is heterogeneously distributed throughout the study area, resulting in differences in variances of disease risks across different areas in the map. Thus, statistical comparisons are needed to identify areas where statistically significant clusters of stroke and MI mortality exist, while taking into account population distribution, to better understand disease disparities. This explains the need to use SEB risk maps as well as spatial scan statistics to identify significant high risk spatial clusters. Moreover, other studies have also indicated that interpreting the results of cluster detection along with the spatial distribution of risk, especially with Bayesian smoothing, can strengthen findings of spatial analysis [68-70].

Spatial scan statistics were used to identify and assess the statistical significance of areas with high risk of stroke and MI clusters. This methodology, implemented in SaTScan 8.0 [71], has many advantages over other cluster detection methods: it corrects for multiple comparisons, adjusts for population heterogeneity in the study area, identifies clusters without a priori specification of their suspected location or size and thus limits pre-selection bias, and allows for adjustment for covariates $[54,72]$. Using visualization of spatial patterns of SEB smoothed risk in conjunction with the results of spatial scan statistics in this study, the neighborhoods with the highest risks were consistent and easy to identify. Detection of spatial clusters of disease allows health planners to effectively identify and plan for the specific characteristics and health needs of the populations with the highest risks of disease $[68,69]$. For instance, median levels of stroke and MI mortality risk were observed for Knox County in the smoothed risk maps, but cluster detection highlighted just a few neighborhoods with statistically significant higher risk than surrounding neighborhoods in the county. The implication is that health planning and programs can be focused to specific neighborhoods of high risk to better meet their health needs instead of using a one-size-fits-all strategy for all neighborhoods within a county. Thus, neighborhood level analysis allows limited resources and efforts to be targeted to the highest risk communities [68].

\section{Conclusion}

Spatial clusters of high mortality risks were identified at the neighborhood level, indicating disparities in risk of death from MI and stroke within counties of the study area. The implication is that, from a needsbased health planning standpoint, a neighborhood level approach is important to ensure that resources and efforts are targeted to the populations most in need. This study also demonstrated that the use of spatial statistics, cluster detection methods, and GIS can aid health planners in appropriately assessing and identifying spatial disparities in risk in populations so as to better guide evidence-based health planning decisions. 


\section{List of Abbreviations Used}

CDC: Centers for Disease Control and Prevention; CT: census tracts; ICD: International classification of diseases; GIS: geographic information systems; MI: myocardial infarction; SEB- spatial empirical Bayes; TN: Tennessee; US: United States.

\section{Acknowledgements}

We extend our appreciation to the Tennessee Department of Health for providing the data and to the American Heart Association Greater Southeast Affiliate for funding this study (grant number 0865290E).

\section{Author details}

${ }^{1}$ The University of Tennessee, Department of Comparative Medicine, 2407 River Drive Knoxville, Tennessee 37996-4543, USA. ${ }^{2}$ East Tennessee State University, Department of Biostatistics and Epidemiology, P.O. Box 70259, Johnson City, TN 37614-1709, USA.

\section{Authors' contributions}

AP was involved in data acquisition, analyses, and interpretation, as well as preparation of the manuscript. TA was involved in data acquisition and review of the manuscript. AO conceived the research idea and was involved in data acquisition, study design, interpretation of results, as well as extensive editing of the manuscript. All authors certify that they have participated sufficiently in the research to believe in its overall validity and have read and approved the final manuscript.

\section{Competing interests}

The authors declare that they have no competing interests.

Received: 9 March 2011 Accepted: 12 August 2011

Published: 12 August 2011

\section{References}

1. Writing Group Members, Lloyd-Jones D, Adams RJ, Brown TM, Carnethon M, Dai S, De Simone G, Ferguson TB, Ford E, Furie K, Gillespie C, Go A, Greenlund K, Haase N, Hailpern S, Ho PM, Howard V, Kissela B, Kittner S, Lackland D, Lisabeth L, Marelli A, McDermott MM, Meigs J, Mozaffarian D, Mussolino M, Nichol G, Roger VL, Rosamond W, Sacco R, et al: Heart Disease and Stroke Statistics-2010 Update: A Report From the American Heart Association. Circulation 2010, 121(7):e46-215.

2. Center for Disease Control and Prevention: Prevalence of Stroke - United States, 2005. MMWR 2007, 56(19):471-474

3. Cooper R, Cutler J, Desvigne-Nickens P, Fortmann SP, Friedman L, Havlik R, Hogelin G, Marler J, McGovern P, Morosco G, Mosca L, Pearson T, Stamler J, Stryer D, Thom T: Trends and disparities in coronary heart disease, stroke, and other cardiovascular diseases in the United States - Findings of the National Conference on Cardiovascular Disease Prevention. Circulation 2000, 102(25):3137-3147.

4. Gillum RF, Ingram DD: Relation between residence in the southeast region of the United States and stroke incidence - The NHANES I Epidemiologic Followup Study. American Journal of Epidemiology 1996, 144(7):665-673.

5. Pickle LW, Mungiole M, Gillum RF: Geographic variation in stroke mortality in blacks and whites in the United States. Stroke 1997 28(8):1639-1647.

6. Rich DQ, Gaziano JM, Kurth T: Geographic patterns in overall and specific cardiovascular disease incidence in apparently healthy men in the United States. Stroke 2007, 38(8):2221-2227.

7. Baldwin LM, MacLehose RF, Hart LG, Beaver SK, Every N, Chan L: Quality of care for acute myocardial infarction in rural and urban US hospitals. Journal of Rural Health 2004, 20(2):99-108.

8. Center for Disease Control and Prevention: Disparities in Deaths from Stroke Among Persons Aged < 75 Years -United States, 2002. MMWR 2005, 54(19):477-504

9. Glymour MM, Avendano M, Berkman LF: Is the 'Stroke Belt' worn from childhood? Risk of first stroke and state of residence in childhood and adulthood. Stroke 2007, 38(9):2415-2421.

10. Halverson J: An analysis of disparities in health status and access to health care in the Appalachian region. The Appalachian Regional Comission Washington D.C.: West Virginia University; 2004.
11. Halverson J, Barnett E, Casper M: Geographic disparities in heart disease and stroke mortality among black and white populations in the Appalachian region. Ethnicity \& Disease 2002, 12:82-91.

12. Centers for Disease Control and Prevention, National Center for Health Statistics: Compressed Mortality File 1999-2006. CDC WONDER On-line Database 2009.

13. Howard G, Cushman M, Prineas RJ, Howard VJ, Moy CS, Sullivan LM, D'Agostino RB, McClure LA, Pulley L, Safford MM: Advancing the hypothesis that geographic variations in risk factors contribute relatively little to observed geographic variations in heart disease and stroke mortality. Preventive Medicine 2009, 49(2-3):129-132.

14. Braveman PA, Cubbin C, Egerter S, Chideya S, Marchi KS, Metzler M, Posner S: Socioeconomic status in health research - One size does not fit all. Jama-Journal of the American Medical Association 2005, 294(22):2879-2888

15. Gerber Y, Weston SA, Killian JM, Therneau TM, Jacobsen SJ, Roger VL: Neighborhood income and individual education: Effect on survival after myocardial infarction. Mayo Clinic Proceedings 2008, 83(6):663-669.

16. Lovasi GS, Moudon AV, Smith NL, Lumley T, Larson EB, Sohn DW, Siscovick DS, Psaty BM: Evaluating options for measurement of neighborhood socioeconomic context: Evidence from a myocardial infarction case-control study. Health \& Place 2008, 14(3):453-467.

17. Petrelli A, Gnavi R, Marinacci C, Costa G: Socioeconomic inequalities in coronary heart disease in Italy: A multilevel population-based study. Social Science \& Medicine 2006, 63(2):446-456.

18. Roux AVD, Merkin SS, Arnett D, Chambless L, Massing M, Nieto FJ, Sorlie P, Szklo M, Tyroler HA, Watson RL: Neighborhood of residence and incidence of coronary heart disease. New England Journal of Medicine 2001, 345(2):99-106.

19. Stjarne MK, Fritzell J, De Leon AP, Hallqvist J: Neighborhood socioeconomic context, individual income and myocardial infarction. Epidemiology 2006, 17(1):14-23.

20. Sundquist J, Malmstrom M, Johansson SE: Cardiovascular risk factors and the neighbourhood environment: a multilevel analysis. International Journal of Epidemiology 1999, 28(5):841-845.

21. Tonne C, Schwartz J, Mittleman M, Melly S, Suh H, Goldberg R: Long-term survival after acute myocardial infarction is lower in more deprived neighborhoods. Circulation 2005, 111(23):3063-3070.

22. Clayton D, L B: Bayesian methods for mapping disease risk. In Geographical and Enivornmental Epidemiology. Edited by: Elliott CJ, English D, Stern R. Oxford: Oxford University Press; 1997:206-220.

23. Waller L, Gotway C: Mapping smoothed rates and probabilites. Applied Spatial Statistics for Public Health Data Hoboken, New Jersey: John Wiley \& sons, Inc:; 2004, 86-104.

24. Pfeiffer DU, Robinson TP, Stevenson M, Stevens KB, Rogers DJ, Clements AC Spatial variation in risk. Spatial Analysis in Epidemiology New York: Oxford Univeristy Press; 2008, 67-80.

25. US Census Bureau: Census Tract and Block Numbering Areas. 200 [http://www.census.gov/geo/www/cen_tract.html], Accessed November 15, 2009.

26. Frenette $M$, Picot $G$, Sceviour R: How long do people live in low-income neighbourhoods? Evidence for Toronto, Montreal and Vancouver. Ottawa, Canada: Statistics Canada; 2004.

27. Odoi A, Wray R, Emo M, Birch S, Hutchison B, Eyles J, Abernathy T: Inequalities in neighbourhood socioeconomic characteristics: potential evidence-base for neighbourhood health planning. International Journal of Health Geographics 2005, 4(20):1-15.

28. Holmstrand P: BatchGeo. 2010 [http://batchgeo.com/], Accessed August 1 2009.

29. Roongpiboonsopit D, Karimi HA: Comparative evaluation and analysis of online geocoding services. International Journal of Geographical Information Science 2010, 24(7):1081-1100.

30. Google Maps Web Services: The Google Geocoding API. 2011 [http://code google.com/apis/maps/documentation/geocoding/], Accessed May 112011.

31. Environmental Systems Research Institute: ArcGIS 9.3 Redlands, CA; 2008.

32. US Census Bureau: Cartographic Boundary File. 2008 [http://www.census. gov/geo/www/cob/bdy_files.html], Accessed November 10, 2008.

33. US Census Bureau: American Fact Finder. 2009 [http://factfinder.census. gov/home/saff/main.html?_lang=en], Accessed November 13, 2009. 
34. Howard G, Howard VJ, Katholi C, Oli MK, Huston S: Decline in US stroke mortality - An analysis of temporal patterns by sex, race, and geographic region. Stroke 2001, 32(10):2213-2218.

35. Casper M, Barnett E, Halverson J, Elmes G, Braham V, Majeed Z, Bloom A Stanley S: Women and heart disease: An atlas of racial and ethnic disparities in mortality. Office for Social Environment and Health Research Morgantown, W: West Virginia University; 1999.

36. Ineid H, Fonarow GC, Cannon CP, Hernandez AF, Palacios IF, Maree AO, Wells Q, Bozkurt B, LaBresh KA, Liang L, Hong Y, Newby LK, Fletcher G, Peterson E, Wexler L, for the Get With the Guidelines Steering Committee and I: Sex Differences in Medical Care and Early Death After Acute Myocardial Infarction. Circulation 2008, 118(25):2803-2810.

37. Morewitz SJ: Cardiovascular Disease. Chronic Diseases and Health Care New York: Springer US; 2006, 139-175.

38. Rose KM, Suchindran CM, Foraker RE, Whitsel EA, Rosamond WD, Heiss G, Wood JL: Neighborhood Disparities in Incident Hospitalized Myocardial Infarction in Four US Communities: The ARIC Surveillance Study. Annals of Epidemiology 2009, 19(12):867-874.

39. Alter DA, Naylor CD, Austin P, Tu JV: Effects of socioeconomic status on access to invasive cardiac procedures and on mortality after acute myocardial infarction. New England Journal of Medicine 1999, 341(18):1359-1367.

40. Tofler GH, Muller JE, Stone PH, Davies G, Davis VG, Braunwald E: Comparison of long-term outcome after acute myocardial-infarction in patients never graduated from high-school with that in more educated patients. American Journal of Cardiology 1993, 71(12):1031-1035

41. Winkleby $M$, Sundquist $K$, Cubbin C: Inequities in CHD incidence and case fatality by neighborhood deprivation. American Journal of Preventive Medicine 2007, 32(2):97-106.

42. Chaix B, Rosvall M, Merlo J: Neighborhood socioeconomic deprivation and residential instability - Effects on incidence of ischemic heart disease and survival after myocardial inforction. Epidemiology 2007, 18(1):104-111.

43. Hart C, Hole D, Smith G: The contribution of risk factors to stroke differentials, by socioeconomic position in adulthood: The Renfrew/ Paisley study. American Journal of Public Health 2000, 90(11):1788-1791.

44. Glymour MM, Kosheleva A, Boden-Albala B: Birth and adult residence in the Stroke Belt independently predict stroke mortality. Neurology 2009, 73(22):1858-1865.

45. Kaplan G, Keil J: Socioeconomic factors and cardiovascular disease: a review of the literature. Circulation 1993, 88:1973-1998.

46. SAS Institute Inc.: SAS Version 9.2. Cary, N.C.; 2007

47. SAS Institute Inc.: SAS OnlineDoc ${ }^{\circledast}$ 9.2. Cary, NC: SAS Institute Inc.; 9.22007.

48. StataCorp: Stata Statistical Software: Release 11 College Station, TX: StataCorp LP; 2009.

49. Anselin L, Syabri I, Kho Y: GeoDa: An Introduction to Spatial Data Analysis. Geographical Analysis 2006, 38(1):5-22.

50. Cuzick J, Elliott P: Small-area studies: purpose and methods. In Geographical and Environmental Epidemiology: Methods for Small-Area Studies. Edited by: Elliott P, Cusick J, English D, Stern R. Oxford: Oxford University Press; 1997:14-21.

51. Kulldorff M, Information Management Services, Inc.: SaTScan v8.0: Software for the spatial and space-time scan statistics 2009

52. Kulldorff M: A spatial scan statistic. Communications in Statistics: Theory and Methods 1997, 26:1481-1496.

53. Dwass M: Modified randomization tests for non-parametric hypothesis. Annals of Mathematical Statistics 1957, 28:181-187.

54. Kulldorff M, Nagarwalla N: Spatial disease clusters: detection and inference. Statistics in Medicine 1995, 14:799-810.

55. Walsh SJ, DeChello LM: Geographical variation in mortality from systemic lupus erythematosus in the United States. Lupus 2001, 10(9):637-646.

56. Winkleby MA, Cubbin C: Influence of individual and neighbourhood socioeconomic status on mortality among black, Mexican-American, and white women and men in the United States. J Epidemiol Community Health 2003, 57(6):444-452.

57. Tennessee Department of Health, Tennessee State University, Center for HealthResearch, University of Tennessee Health Science Center: The Burden of Heart Disease and Stroke in Tennessee. Nasvhille, TN: Tennessee Health of Department; 2006.
58. Liao YL, Greenlund KJ, Croft JB, Keenan NL, Giles WH: Factors explaining excess stroke prevalence in the US stroke belt. Stroke 2009, 40(10):3336-3341.

59. Diez-Roux AV: Bringing context back into epidemiology: Variables and fallacies in multilevel analysis. American Journal of Public Health 1998 88(2):216-222.

60. Franco M, Diez-Roux AV, Nettleton JA, Lazo M, Brancati F, Caballero B, Glass T, Moore LV: Availability of healthy foods and dietary patterns: the Multi-Ethnic Study of Atherosclerosis. American Journal of Clinical Nutrition 2009, 89(3):897-904.

61. Friis R, Sellers T: Epidemiology for Public Health Practice. 3 edition. Sudbury, Massachusetts: Jones and Bartlett Publishers; 2003.

62. Lloyd-Jones D, Martin D, Larson M, Levy D: Accuracy of death certificates for coding coronary heart disease as the cause of death. Annals of Internal Medicine 1998, 129(12):1020-1026.

63. Bithell J: A classification of disease mapping methods. Statistics in Medicine 2000, 19:2203-2215.

64. Odoi A, Martin SW, Michel P, Holt J, Middleton D, Wilson J: Geographical and temporal distribution of human giardiasis in Ontario, Canada. 2003, 2(1):5.

65. Rogerson P, Yamada I: Statistical Detection and Surveillance of Geographic Clusters Boca Raton, FL: Taylor \& Francis Group, LLC; 2009.

66. Dohoo I, Martin W, Stryhn H: Analysis of spatial data: Introduction and visualisation. In Veterinary Epidmiologic Research. Edited by: McPike SM. Charlottetown, Canada: VER Inc:; 2009:

67. Jenks $\mathrm{G}$ : The data model concept in statistical mapping. The International Yearbook of Cartography 1967, 7:186-190.

68. Holowaty EJ, Norwood TA, Wanigaratne S, Abellan JJ, Beale L: Feasibility and utility of mapping disease risk at the neighbourhood level within a Canadian public health unit: an ecological study. International Journal of Health Geographics 2010, 9.

69. Odoi A, Martin SW, Michel P, Middleton D, Holt J, Wilson J: Investigation of clusters of giardiasis using GIS and a spatial scan statistic. Int J Health Geogr 2004, 3:11.

70. Tango T: A test for spatial disease clustering adjusted for multiple testing. Statistics in Medicine 2000, 19:191-204.

71. Kulldroff M, Information Management Services, Inc.: SaTScan v8.0: Software for the spatial and space-time scan statistics 2009.

72. Kulldroff M: A spatial scan statistic. Communications in Statistics: Theory and Methods 1997, 26:1481-1496.

\section{Pre-publication history}

The pre-publication history for this paper can be accessed here: http://www.biomedcentral.com/1471-2458/11/644/prepub

doi:10.1186/1471-2458-11-644

Cite this article as: Pedigo et al:: Neighborhood disparities in stroke and myocardial infarction mortality: a GIS and spatial scan statistics approach. BMC Public Health 2011 11:644.

\section{Submit your next manuscript to BioMed Central and take full advantage of:}

- Convenient online submission

- Thorough peer review

- No space constraints or color figure charges

- Immediate publication on acceptance

- Inclusion in PubMed, CAS, Scopus and Google Scholar

- Research which is freely available for redistribution

Submit your manuscript at www.biomedcentral.com/submit
C Biomed Central 